\title{
Third-order fixed-ratio schedules of reinforcement '
}

GEORGE BIGELOW and TRA VIS THOMPSON, Departme'nt of Psychiatry, University of Minnesota. Minmeapolis, Minn. 55455

Third-order fixed-ratio schedules of reinforcement were examined with both rats and monkeys. Two stimuli were briefly presented in different relations to primary reinforcement: $F R$ × /FR y (FR z: $\left.S_{1}\right): S_{2} /$. These stimuli were found effectively to control the pattern of operant performance. with stimuli separated by many responses being more effective in separating rums of responses than were stimali separated by fewer responses. In addition, interactions were foumd between the two schedules of stimulus presentation.

The technique of higher-order reinforcement scheduling has received increasing attention recently. This technique consists of taking the sequence of behavior specified by some schedule contingency, treating this sequence as a unitary response and reinforcing this larger unit on some schedule. Typically, some brief stimulus change occurs following completion of each of these larger response units. This stimulus change appears to serve a conditioned reinforcing function (Findley \& Brady, 1965). Most earlier work has dealt with FI schedule units which are treated as the basic response in second-order schedules (Kelleher, 1966; deLorge, 1967). The present experiments examine third-order schedules involving fixed-ratio units. In these schedules, two different brief stimulus changes are programmed, each according to a different FR schedule. The basic schedule studied can be notated as FR x [FR y (FR z: $S_{1}$ ): $S_{2}$ l, where each completion of an FR $z$ unit results in an $S_{1}$ presentation, each $y$-th presentation of $S_{1}$ results in an $S_{2}$ presentation, and each $x$-th presentation of $S_{2}$ results in primary reinforcement. The total number of responses required for primary reinforcement is $x$ times $y$ times $z$. The total number of $S_{1}$ presentations required is $x$ times $y$, and the total number of $S_{2}$ presentations required is $x$.

The use of higher-order schedules involving more than one type of stimulus change allows the simultaneous investigation of several schedules of stimulus presentation, and also allows for the investigation of possible interactions between these schedules of stimulus presentations.

In the two experiments to follow, data were recorded as the median time required to complete each of the component FR $z$ units involved in the overall schedule. In the figures the time to complete the initial FR $z$ unit is omitted since it includes the post-reinforcement pause and therefore greatly exceeds the scale used. Arrows on the figures indicate those components followed by $S_{2}$ presentations $\left(S_{2}\right.$ presentations also occurred at primary reinforcement, but are not indicated).

\section{EXPERIMENT 1}

Two adult male rhesus monkeys, approximately $15 \mathrm{~kg}$ each, served as Ss. Each was housed in a steel mesh cage which served as both the living and the experimental space. Each cage was fitted with a stimulus panel, a Lindsley manipulandum, and a food hopper to which reinforcements were delivered (each reinforcement consisted of six Purina Monkey Chow biscuits). Electromechanical programming equipment was located in a separate room.

The basic schedule used consisted of a total of 480 responses per reinforcement, arranged as a sequence of 12 consecutive FR 40 units. Using this constant response requirement, three different schedules of stimulus presentation between primary reinforcements were studied. The stimuli used were illumination of the feeder hopper $\left(S_{1}\right)$ and a tone presentation $\left(S_{2}\right)$. Both stimuli were of 6 -sec duration.

Figure 1 shows the performance of one of the Ss on the first two schedules used. The initial schedule was FR 2 [FR 6 (FR 40: $S_{1}$ ): $S_{2}$ \}, and programmed an $S_{1}$ presentation following each of the first 11 FR 40 units, and an $S_{2}$ presentation

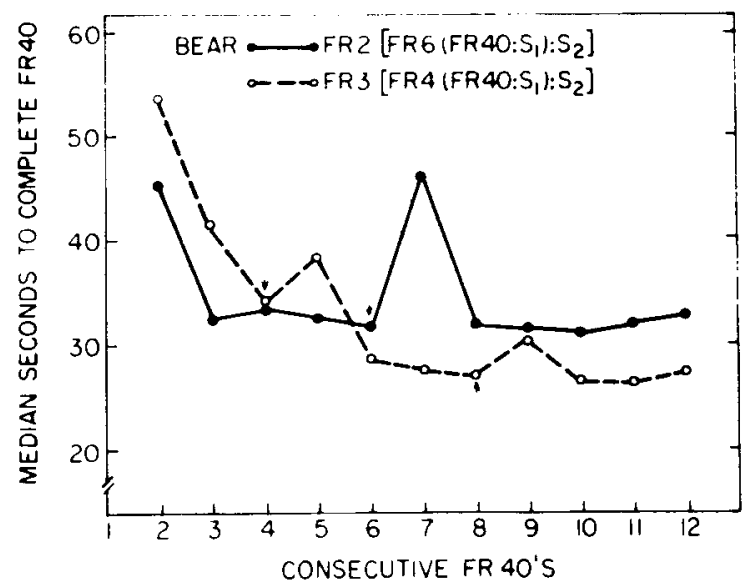

Fig. 1. Median time to complete successive Fixed Ratio-40 components of two third order schedules by a Rhesus monkey. The total number of responses per food reinforcement remained constant, while the number of FR-40 units leading to $S_{1}$ (illumination of feeder hopper) and $S_{2}$ (tone) varied.

following each sixth FR 40 unit. Since $S_{1}$ was not presented following the terminal FR 40 , it was never directly paired with primary reinforcement. $S_{2}$ did directly precede primary reinforcement. In the second schedule, FR 3 [FR 4 (FR 40: $S_{1}$ ): $S_{2}$ ], the schedule of presentation of $S_{1}$ was unaltered, but now $S_{2}$ was presented more frequently-after each fourth FR 40 unit. For both of these schedules, Fig. 1 shows that the components following $S_{2}$ presentations require considerably longer to complete than do those components preceding $S_{2}$ presentations. In addition, the time increment produced by $S_{2}$ presentation is less when three $S_{2}$ presentations are required for primary reinforcement than when only two $S_{2}$ presentations are required. The second $S$ showed essentially the same result.
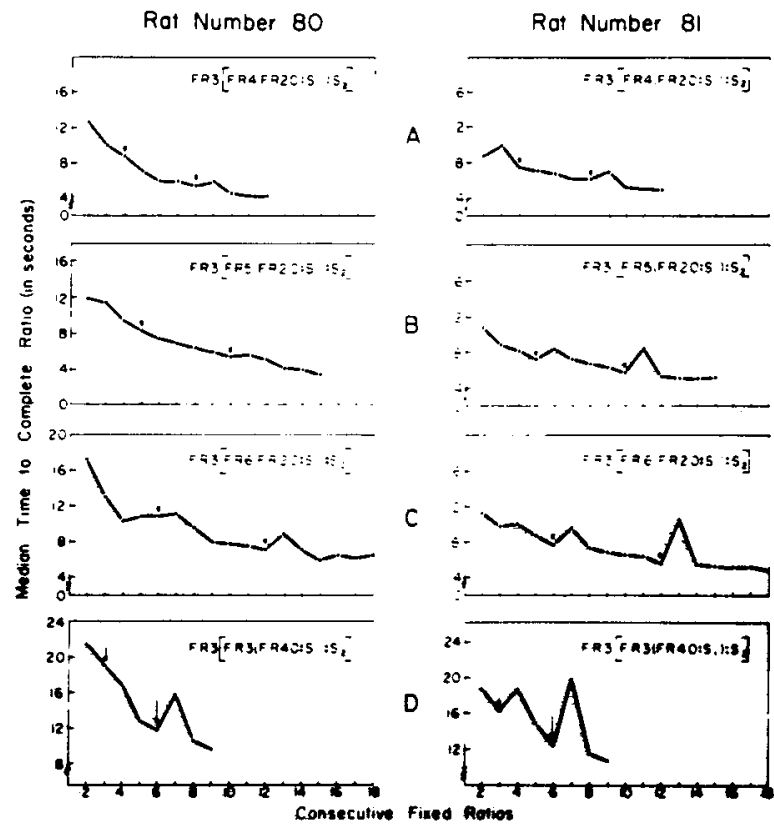

Fig. 2. Median time to complete successive frst order FR components of third order fixed ratio schedules by two rats. $A, B$ and $C$ how the effect of increasing the number of $F R 20$ components for each $S_{2}$. D shows the effect of doubling the size of the first order component. 
Rot Number 80
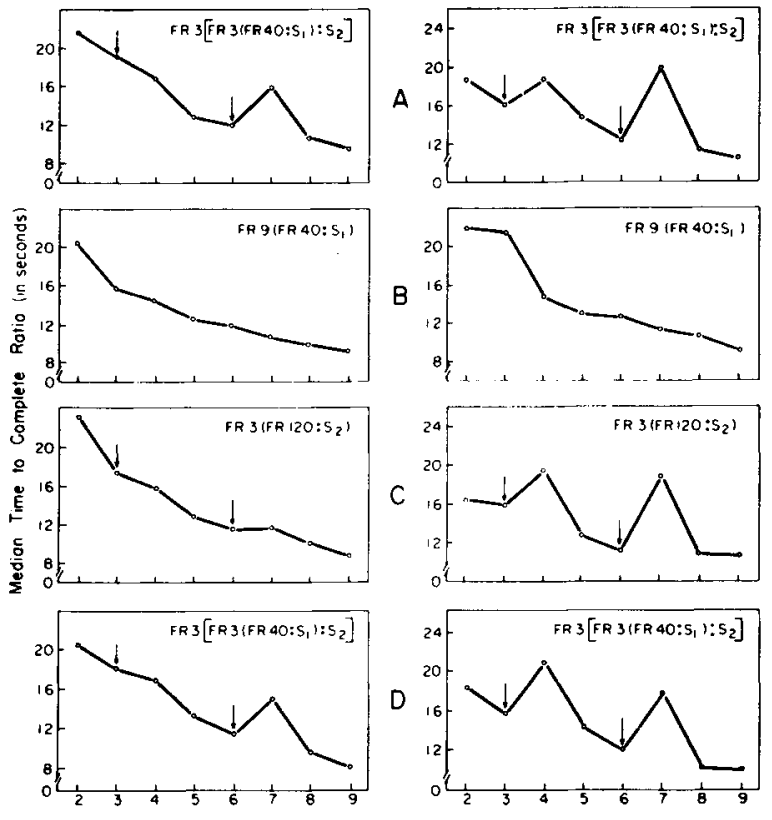

Consecutive Fixed Ratios

Fig. 3. Median time to complete successive first order FR components of a third order schedule (A), and a second order schedule with the same total requirement (B), and a second order schedule. with a first order schedule three times as large having the same total responses per food reinforcement (C). D replicates A showing the reliability of the phenomenon.

In the third schedule used, the presentations of $S_{1}$ were discontinued. This schedule was FR 3 [FR 160: $S_{2}$ ]. This produced no substantial deviation from the pattern of performance on FR 3 [FR 4 (FR 40: $S_{1}$ ): $S_{2}$ ], and the data are therefore not reproduced.

\section{EXPERIMENT 2}

Two male Sprague-Dawley albino rats, maintained at $80 \%$ of their free-feeding weights, served as Ss. Each $\mathrm{S}$ was run in a separate standard operant conditioning chamber for sessions of 3 to $4 \mathrm{~h}$. Each reinforcement consisted of five $45 \mathrm{mg}$ Noyes food pellets. Electromechanical programming equipment was located in a separate room. In this experiment, as in the previous one, the reinforcement schedule consisted of a sequence of several consecutive fixed-ratio components, and various patterns of stimulus presentation between primary reinforcement were investigated. Stimulus presentations were of $1 / 2 \mathrm{sec}$ duration; illumination of the feeder hopper served as $S_{1}$, and a tone presentation served as $S_{2}$. In th is experiment, both $S_{1}$ and $S_{2}$ directly preceded primary reinforcement; however, they differed in the number of stimulus presentations preceding primary reinforcement.

The first series of schedules investigated the effects of increasing the number of FR 20 units between $S_{2}$ presentations. The initial schedule was FR 3 [FR 4 (FR 20: $S_{1}$ ): $S_{2}$ ], and programmed primary reinforcement following the third $S_{2}$ presentation; $S_{2}$ presentations were separated by four FR 20 units (and, therefore, four $S_{1}$ presentations). The number of FR 20 units between $S_{2}$ presentations was increased first to five-FR 3 [FR 5 (FR 20: $\left.S_{1}\right): S_{2}$ ] -and then to six-FR 3 [FR 6(FR 20: $\left.S_{1}\right): S_{2}$ ]

Performance on these three schedules is depicted for both animals in Figs. $2 \mathrm{~A}, \mathrm{~B}$, and $\mathrm{C}$. As increasing numbers of responses and $S_{1}$ presentations intervened between $S_{2}$ presentations, the effect of $S_{2}$ presentations in decreasing the rate of the succeeding FR 20 component was increased. This effect could be due either to the increased response requirement or to the increased number of $S_{1}$ presentations. In the next schedule-FR 3 [FR 3 (FR 40: $S_{1}$ ): $S_{2}$ ] -shown in Fig. 2D, the number of responses intervening between $S_{2}$ presentations remained the same as in Fig. 2C, but now only one half as many $S_{1}$ presentations occurred, as the basic component unit was increased from FR 20 to FR 40. Comparing these two schedules, in Fig. 2C and D, reveals some indication of an interaction between $S_{1}$ and $S_{2}$. The increment in time to complete the component following $S_{2}$ is greater in Fig. 2D where $S_{1}$ is presented relatively infrequently than in Fig. $2 \mathrm{C}$ where $S_{1}$ is presented more frequently. Thus, decreasing the frequency of $S_{1}$ appears to accentuate the effect of $S_{2}$. This comparison is not based upon the absolute time values to complete the components, since the two schedules deal with different size units, but is based upon the differences between the times to complete the component preceding the $S_{2}$ presentation and the component following the $S_{2}$ presentation.

Figure 3 compares the performances generated by the third-order schedule FR 3 [FR 3 (FR 40: $S_{1}$ ): $S_{2}$ ] with those generated by comparable second-order schedules involving presentation of only one of the two stimuli. Figure $3 \mathrm{~A}$ shows the pattern of performance on the baseline third-order schedule, with the $S_{2}$ presentations resulting in an increment in time required to complete the following FR 40 unit. In Fig. 3B, the $\mathrm{S}_{2}$ presentations have been discontinued, resulting in the second-order schedule FR 9 (FR 40: $S_{1}$ ) which generates a monotonic acceleration of response rate across the nine FR 40 units. In Fig. 3C, $\mathrm{S}_{1}$ is discontinued resulting in the secondorder schedule FR 3 (FR 120: $S_{2}$ ). For Rat No. 80 this reveals an interaction between the two stimuli used. Without $S_{1}$ presentations, $S_{2}$ has only a very slight effect upon the time required to complete the following component. Omission of $S_{1}$ had essentially no effect for Rat No. 81. In Fig. 3D, the performance of the baseline third-order schedule is regained. DISCUSSION

Previous experimenters have shown that brief stimuli presented in second-order schedules can both serve as conditioned reinforcers and control the pattern of operant performance (Findley \& Brady, 1965; Kelleher, 1966; deLorge, 1967). The present experiments do not offer data relevant to the problem of conditioned reinforcement, but they do extend to thirdorder schedules the finding that brief stimulus presentations may control the pattern of performance. This control is exhibited as an increase in the time required to complete the FR unit following the brief stimulus presentation. Examinations of cumulative records revealed that this time increment was due to both a period of pausing and a period of intermediate rate similar to the warm-up often seen at the beginning of long FRs.

The present studies have found that the effectiveness of brief stimulus presentations in controlling the pattern of performance is affected by the number of responses intervening between stimulus presentations, with stimuli separated by many responses being more effective in separating runs of responses than stimuli separated by fewer responses.

In addition, it has been found that in third-order schedules, where more than one brief stimulus is used, there may be interactions between the schedules controlling these different stimuli. In Experiment 2, decreasing the number of $S_{1}$ presentations from 18 to nine increased the power of $S_{2}$; however, decreasing the number of $S_{1}$ presentations to zero decreased the power of $S_{2}$. Further investigation is required to illuminate the factors controlling these interactions, but the existence of interactions suggests that third-order schedules may have properties not readily predictable from second-order schedules.

\section{REFERENCES}

deLORGE, J. Fixed-interval behavior maintained by conditioned reinforcement. Journal of the Experimental Analysis of Behavior, $1967,10,271-276$.

FINDLEY, J. D., \& BRADY, J. V. Facilitation of large ratio performance by use of conditioned reinforcement. Journal of the Experimental Analysis of Behavior, 1965, 8, 125-129.

KELLEHER, R. T. Chaining and conditioned reinforcement. In W. Honig (Ed.), Operant behavior: Areas of research and application. New York: Appleton-Century-Crofts, 1966.

NOTE

1. This research was supported in part by U.S.P.H.S. Research Grant MH-11135 to the University of Minnesota. 